\title{
Preparation and in vitro evaluation of a biomimetic nanoscale calcium phosphate coating on a polyethylene terephthalate artificial ligament
}

\author{
CHEN CHEN $^{1 *}$, HONG LI ${ }^{2 *}$, CHANGAN GUO ${ }^{1}$ and SHIYI CHEN ${ }^{2}$ \\ ${ }^{1}$ Department of Orthopedics, Zhongshan Hospital, Shanghai 200032; \\ ${ }^{2}$ Department of Sports Medicine, Huashan Hospital, Shanghai 200040, P.R. China
}

Received May 28, 2014; Accepted January 23, 2015

DOI: $10.3892 /$ etm.2016.3269

\begin{abstract}
In the present study, a polyethylene terephthalate (PET) artificial ligament was coated with an organic layer-by-layer (LBL) self-assembled template of chitosan and hyaluronic acid, and then incubated in a calcium phosphate $(\mathrm{CaP})$ solution to prepare a biomimetic CaP coating. The surface characterization of the ligament was examined using scanning electron microscopy, atomic force microscopy and energy-dispersive X-ray spectroscopy. The effects of $\mathrm{CaP}$ coatings on the osteogenic activity of MC3T3 E1 mouse osteoblastic cells were investigated by evaluating their attachment, proliferation and the relative expression levels of alkaline phosphatase. The results revealed that the organic LBL template on the PET artificial ligament was effective for $\mathrm{CaP}$ apatite formation. Following incubation for $72 \mathrm{~h}$, numerous nanoscale $\mathrm{CaP}$ apatites were deposited on the PET ligament fibers. In addition, the results of the in vitro culture of MC3T3-E1 mouse osteoblastic cells demonstrated that the CaP coating had a good biocompatibility for cell proliferation and adhesion, and the CaP-coated group had a significantly higher alkaline phosphatase activity compared with the uncoated control group after seven days of cell culture. Collectively, these results demonstrated that the biomimetic nanoscale CaP-coated PET artificial ligaments have potential in bone-tissue engineering.
\end{abstract}

Correspondence to: Dr Shiyi Chen, Department of Sports Medicine, Huashan Hospital, 12 Wulumuqi Zhong Road, Shanghai 200040, P.R. China

E-mail: sychen11@163.com

Dr Changan Guo, Department of Orthopedics, Zhongshan Hospital, 180 Femling Road, Shanghai 200032, P.R. China

E-mail: changanguo1@hotmail.com

${ }^{*}$ Contributed equally

Key words: surface modification, calcium phosphate, biomimetic mineralization, polyethylene terephthalate, artificial ligament

\section{Introduction}

Polyethylene terephthalate (PET) artificial ligaments have been used for a number of years as biomedical implants in humans, including in the ligament advanced reinforcement system (LARS ${ }^{\oplus}$; Surgical Implants and Devices, Arc-sur-Tille, France) $(1,2)$. With non-degradable features, PET ligaments remain highly mechanically stable for a long time. However, PET artificial ligaments also have a hydrophobic character and chemical inertness, which limit the healing with the surrounding bone following implantation in the host bone $(3,4)$. Instead of a normal tendon-bone fibrocartilaginous insertion, a weak fibrous scar tissue band occurs between the pure PET ligament graft and the host bone (5). In a multicenter follow-up study, Gao et al (6) observed fibrous tissue between the PET artificial ligament graft and the bone in the second-look arthroscopy of several failed cases. These observations indicated that the surface property of the PET graft played a crucial role in graft-bone healing in vivo. Therefore, surface modification on the PET ligament is required in biomedical fields to improve and promote the graft-bone healing of PET artificial ligaments following implantation.

Currently, surface coating with calcium phosphate (CaP) or hydroxyapatite (HAp) is widely applied as a surface modification of metal implants in order to promote the graft-bone healing (7-9). A CaP coating on grafts is able to enhance osteoblast differentiation, provide an ideal environment for osteoblast activity and stimulate bone formation at the interface between the graft and bone. Bigi et al (10) used a biomimetic approach to obtain a nanocrystalline HAp coating on metallic substrates and demonstrated that the HAp coating on a metallic alloy was able to promote bone ingrowth around grafts.

The attachment of a native anterior cruciate ligament (ACL) on the bone can be divided into four zones histologically, which include the ligament, unmineralized fibrocartilage, mineralized fibrocartilage and bone, and this composite structure can sustain a very high load without failure (11). For a pure tendon or artificial ligament graft, forming this type of mineralized composite structure at the interface between graft and bone can be a challenge. Thus, $\mathrm{CaP}$ has also been applied to enhance 
the tendon healing of ACL grafts in the bone tunnel $(12,13)$. In an animal study (14), CaP-hybridized tendons were shown to regenerate a direct insertion-like formation of tendons, similar to that of a normal healthy ACL insertion, at three weeks postoperatively. The coating of CaP on a PET artificial ligament to enhance tendon-to-bone insertion site formation has subsequently been attracting increasing interest.

Previously, nanoscale HAp particles have been coated on a PET artificial ligament to enhance graft-bone healing, and it was demonstrated that the HAp coating had a positive effect on the induction of graft healing within the bone tunnel (15). However, an agglomeration phenomenon of these nano HAp particles on the PET graft was observed, and the distribution of the coating was not as uniform as desired. In the current study, apatite deposition via biomimetic mineralization (BM) was attempted through a layer-by-layer (LBL) self assembling organic template on the PET ligament. The aim of the present study, therefore, was to modify the PET ligament with a nanoscale biomimetic $\mathrm{CaP}$ coating and evaluate the effect of the coating in vitro. The LBL organic template was hypothesized to induce a nanoscale CaP coating deposition on the graft. Furthermore, this CaP coating was hypothesized to stimulate the activity of MC3T3-E1 osteoblastic cells in vitro.

\section{Materials and methods}

Preparation of PET sheets. PET sheets from a LARS ligament were removed and immersed in $75 \%$ alcohol solution for $4 \mathrm{~h}$ to eliminate any impurities. The sheets were subsequently washed with copious quantities of deionized water and dried under reduced pressure at $37^{\circ} \mathrm{C}$ for $24 \mathrm{~h}$.

Preparation of $L B L$ sheets via an LBL self-assembly process. The $\gamma$-methacryloxypropyltrimethoxysilane (ACROS Organics, Geel, Belgium) was grafted on the cleaned PET sheets using plasma surface modification (CTP-2000 K plasma apparatus; Nanjing Suman Electronics Co., Ltd. Nanjing, China). The silanized ligament sheets were immersed in a $0.1 \mathrm{wt} \%$ ethanol solution of 3-aminopropyltriethoxysilane (ACROS Organics) for $4 \mathrm{~h}$ at $37^{\circ} \mathrm{C}$ (Fig. 1A), treated with $1 \%$ hydrochloric acid solution for $2 \mathrm{~h}$ at room temperature and subsequently placed for $1 \mathrm{~min}$ into a solution comprising $0.1 \mathrm{wt} \%$ acetic acid and $0.1 \mathrm{wt} \%$ chitosan (CS; Sigma-Aldrich, St. Louis, MO, USA). The sheets were then dipped in $0.1 \mathrm{wt} \%$ hyaluronic acid solution (CPN Spol s.r.o., Dolní Dobrouĉ, Czech Republic) for $1 \mathrm{~min}$ (Fig. 1B). The deposition process was repeated until 10 bilayers of CS and hyaluronic acid had been prepared. Finally, the samples were dried at $37^{\circ} \mathrm{C}$ for $48 \mathrm{~h}$.

BM. CaP solution was prepared by dissolving $\mathrm{CaCl}_{2}(5 \mathrm{mmol})$, $\mathrm{NaH}_{2} \mathrm{PO}_{4}(2 \mathrm{mmol})$ and $\mathrm{NaHCO}_{3}(1.5 \mathrm{mmol})$ salts into 1 litre demineralized water. The LBL-modified sheets were soaked in the solution at $37^{\circ} \mathrm{C}$ for three days to reproduce bone-like apatite on the ligament sheets (Fig. 1C). The BM sheets were subsequently washed gently with distilled water and dried at $30^{\circ} \mathrm{C}$ under reduced pressure for $12 \mathrm{~h}$.

Scanning electron microscopy (SEM). Surface morphologies of the PET sheets, with or without modification, were imaged using SEM (VEGA3; Tescan Co., Ltd., Brno, Czech Republic). Briefly, small sections of the sheets were vacuum-coated with gold (JS-1600; Beijing HTCY Technology, Beijing, China), placed in the vacuum chamber of the SEM and viewed at a $20-\mathrm{kV}$ accelerating voltage.

Atomic force microscopy (AFM). Morphologies of the modified-sheet fibers were studied using AFM in tapping mode (Multimode Nanoscope; Veeco Process Equipment, Inc., Camarillo, CA, USA) using a silicon tip (NSC11/AIBS, Ultrasharp $\mu$ masch; MikroMasch, Lady's Island, SC, USA) under ambient conditions. Scans of $1 \mathrm{x} 1 \mu \mathrm{m}$ areas on the fiber surface were investigated.

Energy-dispersive $X$-ray spectroscopy (EDX). An EDX system (QUANTA X400; Bruker Corporation, Ettlingen, Germany) was used to analyze the elements of the surface modification and measure the ratio of calcium $(\mathrm{Ca})$ and phosphorus $(\mathrm{P})$ on the surface of the mineralized sheets.

In vitro analysis with MC3T3-E1 mouse osteoblastic cells. In vitro experiments were performed with an MC3T3-E1 mouse osteoblastic cell line (Riken Cell Bank, Ibaraki, Japan). The cells were grown in tissue culture polystyrene flasks containing Dulbecco's modified Eagle's medium (Hyclone; GE Healthcare, Logan, UT, USA), supplemented with $15 \%$ fetal bovine serum, $300 \mathrm{mg} / \mathrm{ml} \mathrm{L-glutamine,}$ $100 \mathrm{IU} / \mathrm{ml}$ penicillin and $25 \mathrm{mg} / \mathrm{ml}$ streptomycin solution, at $37^{\circ} \mathrm{C}$ in a humidified atmosphere containing $5 \% \mathrm{CO}_{2}$. The CaP-coated sheets (BM group) and the uncoated sheets (control group), with a 1-cm diameter, were soaked in $70 \%$ ethanol for $30 \mathrm{~min}$ and left overnight in a laminar-flow cabinet to dry. The sheets were washed twice with sterile phosphate-buffered saline (PBS), transferred to a 24-well untreated plate, and incubated at $37^{\circ} \mathrm{C}$ in a humid atmosphere with $5 \% \mathrm{CO}_{2}$ for $4 \mathrm{~h}$ in basic cell culture medium. Following the removal of the medium, cells at the logarithmic growth phase were cultured at $5 \times 10^{4}$ cells $/ \mathrm{ml}$ in the sheets. The sheets were incubated at $37^{\circ} \mathrm{C}$ in a humidified $\mathrm{CO}_{2}$ atmosphere for a period of up to seven days.

Cell proliferation was assessed using a methylthiazol tetrazolium (MTT) assay (Sigma-Aldrich). After the cells had been cultured for 1,3 and 7 days, they were mixed with $200 \mu \mathrm{l}$ complete medium and $20 \mu \mathrm{l}$ MTT solution $(5 \mathrm{mg} / \mathrm{ml}$ in PBS) and incubated at $37^{\circ} \mathrm{C}$ to form MTT formazan crystals. After 4 hours, $200 \mu$ l dimethyl sulfoxide (DMSO) was added, in order to dissolve the formazan crystals. The solution was agitated until it became homogeneous ( $15 \mathrm{~min}$ on a shaker). The optical density (OD) was measured at $570 \mathrm{~nm}$ against a DMSO solution blank using a microplate reader (VICTOR $\mathrm{X}$, PerkinElmer, Inc., Waltham, MA, USA). Three parallel replicates were evaluated for each sample. In addition, the morphology of the MC3T3-E1 cells cultured on the sheets was observed by SEM (VEGA3; Tescan Co., Ltd.) at a 5-kV accelerating voltage. Alkaline phosphatase (ALP) is an enzyme that can be used to indicate the occurrence of active bone formation, since ALP is a byproduct of osteoblast activity. Thus, ALP activity was assessed biochemically on day seven using an ALP testing kit (Houbio Tech Co., Ltd., Hong Kong, China). A $1,270-\mu 1$ solution, containing $250 \mu \mathrm{l}$ priming solution, $20 \mu \mathrm{l}$ 
A

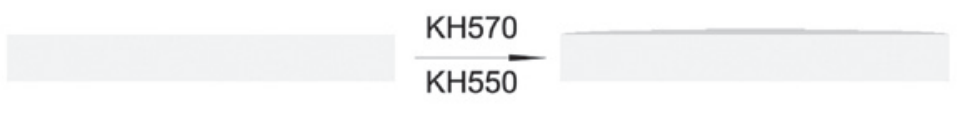

B

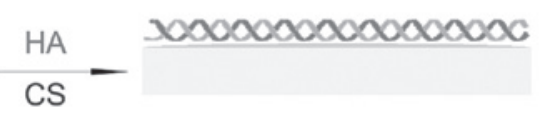

C

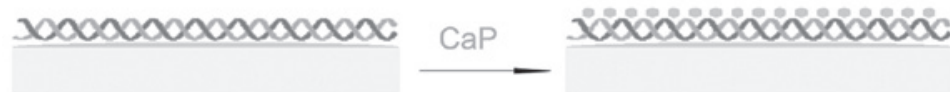

Figure 1. Schematic diagram of the biomimetic mineralization. (A) The polyethylene terephthalate sheet was grafted with KH570 and KH550 through plasma surface modification. (B) The sheet was prepared though a layer-by-layer self assembly process in order to graft an organic template consisting of CS and HA. (C) CaP particles were deposited on the surface of the sheet though a biomimetic process. KH570, $\gamma$-methacryloxypropyltrimethoxysilane; KH550, $\gamma$-aminopropyltriethoxysilane; CS, chitosan; HA, hyaluronic acid; CaP, calcium phosphate.

A

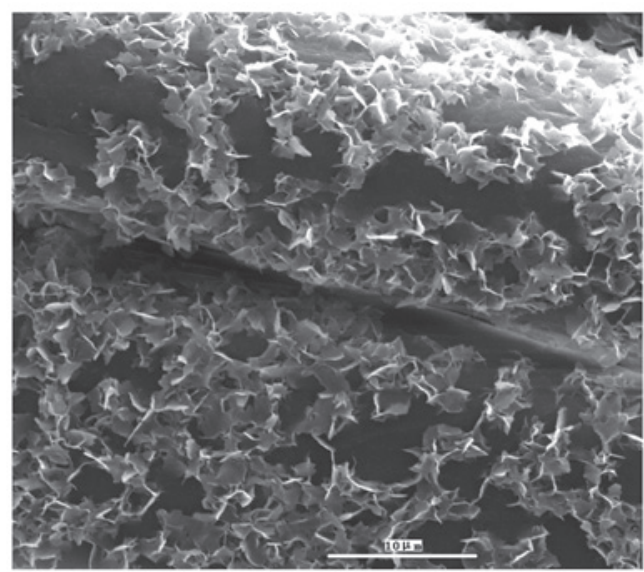

B

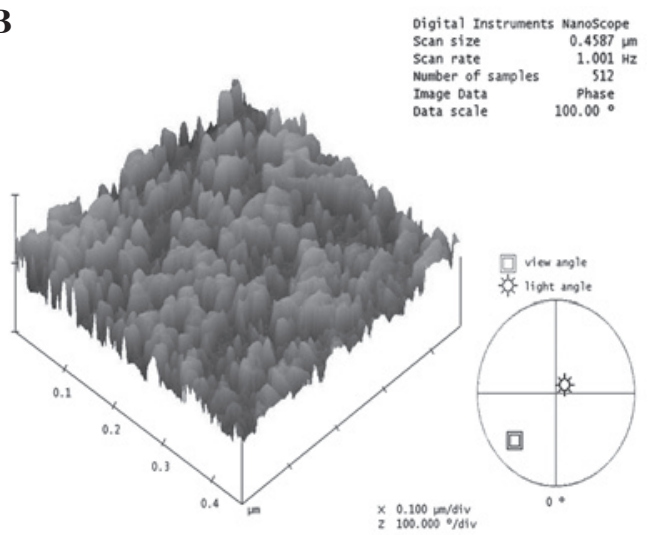

$100726-x 66-22.023$

Figure 2. (A) Scanning electron microscopy characterization. Calcium phosphate (CaP) particles on the biomimetic mineralization (BM) fibers appear in nanoscale (scale bar, $10 \mu \mathrm{m}$ ). (B) Atomic force microscopy (AFM) results of the surface of a BM sheet. On the BM sheet fiber surface, the CaP particle size was $\sim 50 \mathrm{~nm}$ in diameter on the AFM three-dimensional renderings of the mineralized fiber.

sample solution and 1,000 $\mu 1$ PBS, was used for measuring the absorbance OD value of the $p$-nitrophenol at $405 \mathrm{~nm}$ with a microplate reader (UV1000; Shanghai Tian Mei Scientific Instrument Co., Ltd., Shanghai, China).

Statistical analysis. Results are presented as the mean \pm standard deviation. A paired Student's t-test was used to determine statistically significant differences among the groups. Statistical analyses were conducted using Stata 10.0 software (StatCorp LP, College Station, TX, USA). P<0.05 was considered to indicate a statistically significant difference.

\section{Results and Discussion}

As shown in Fig. 2A, the PET grafts were initially smooth and uniform and the LBL sheets appeared smooth and dark. Following immersion in the $\mathrm{CaP}$ solution for three days, numerous CaP particles were deposited on the surface of the PET ligament fibers. In the present study, a previously devised LBL procedure was applied to successfully coat the PET sheets with an organic multilayered template of CS and hyaluronic acid (16). Representative three-dimensional renderings of the mineralized fibers revealed that the fibers

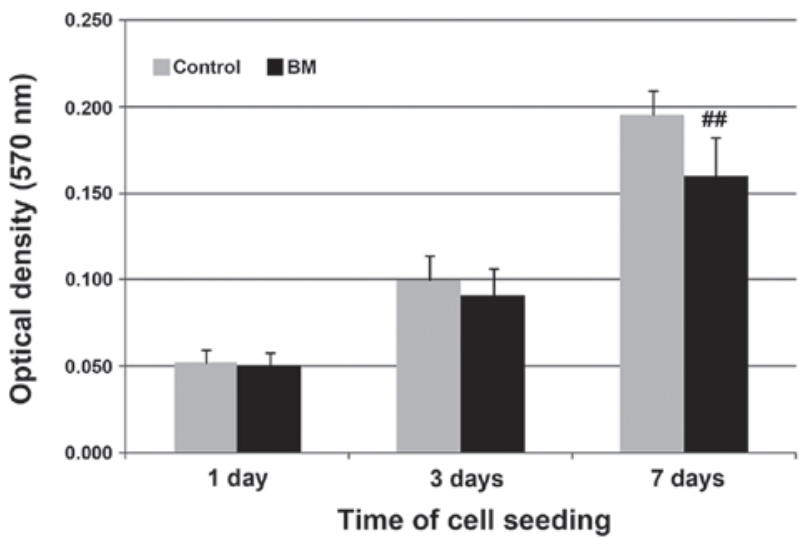

Figure 3. MTT assay results showing MC3T3-E1 cell proliferation on the control and BM sheets. The optical density value increased with time in the two groups. ${ }^{\# \#} \mathrm{P}<0.05$, vs. control (untreated polyethylene terephthalate sheet). $\mathrm{BM}$, biomimetic mineralization.

of the $\mathrm{CaP}$ group exhibited a non-spiky plateau surface (Fig. 2B). When CaP was deposited on the fibers, the particle size was $\sim 50 \mathrm{~nm}$ in diameter. These observations indicated that BM through an organic template was a successful method for producing nanoscale particles on PET fibers. 

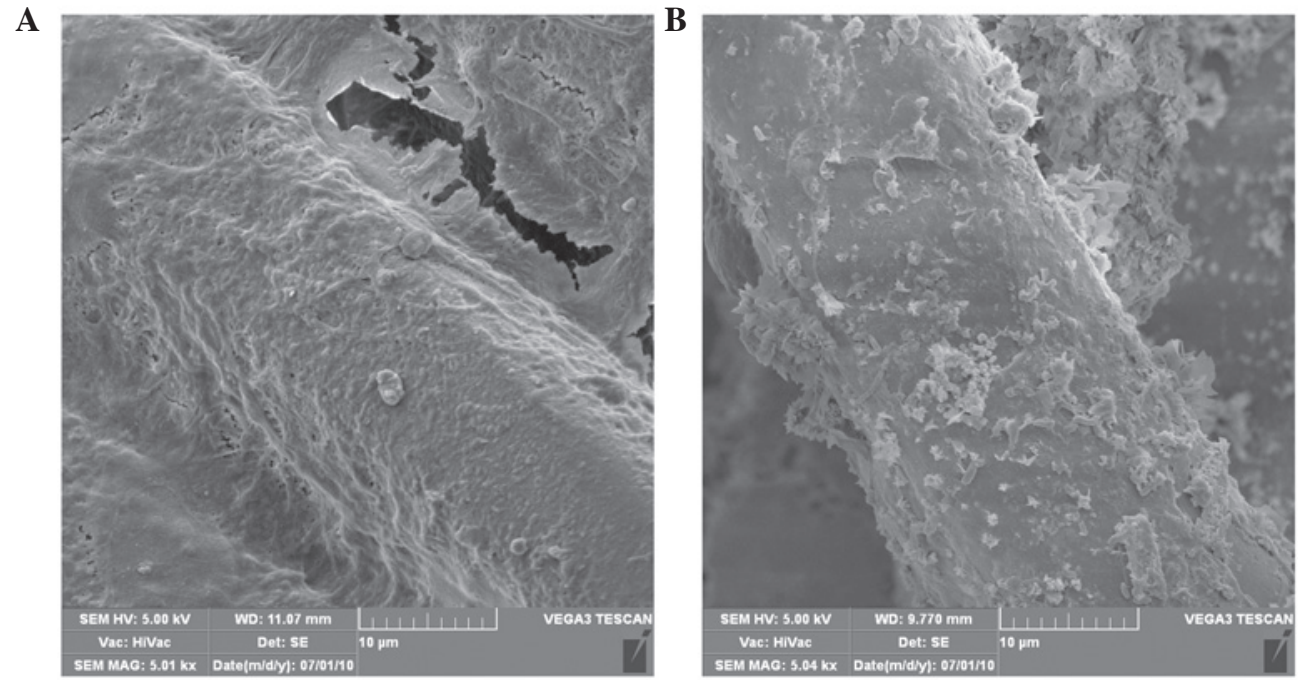

Figure 4. Scanning electron microscopy images of the MC3T3-E1 cells following seeding for seven days on the (A) control group sheets and (B) biomimetic mineralization group sheets.

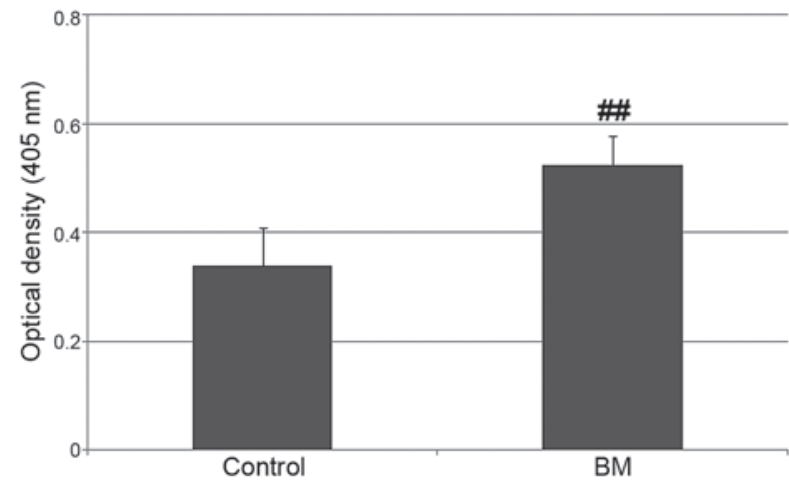

Figure 5. Alkaline phosphatase (ALP) activity of the MC3T3-E1 cells seeded on the control and BM sheets after seven days of culturing. The BM group exhibited significantly higher ALP activity levels compared with the control group. ${ }^{\# \#} \mathrm{P}<0.05$, vs. control (untreated polyethylene terephthalate sheet). BM, biomimetic mineralization.

With numerous carboxylate (-COO-) and amino $\left(-\mathrm{NH}^{3+}\right)$ groups, this organic multilayered template is able to regulate biomineral formation and overcome the dispersion problem of CaP particles on PET fibers (17). In addition, the carboxylic groups interact with the calcium ions of $\mathrm{CaP}$, which enables the multilayered template to effectively stimulate BM and induce the deposition of crystalline $\mathrm{CaP}(18-20)$. However, in contrast to other fast methods for biomimetic deposition of nanocrystalline apatite, the current technique requires approximately three days to deposit a uniform nanoscale $\mathrm{CaP}$ coating on the PET ligament, which may be due to the chemical and physical properties of the surface of the PET ligament.

Through elemental analysis using EDX, the quality ratio of $\mathrm{Ca}$ and $\mathrm{P}$ was determined to be 1.60. Previously, the $\mathrm{Ca} / \mathrm{P}$ ratios of $\mathrm{HAp}$, tricalcium phosphate and octacalcium phosphate have been reported as $1.67,1.50$ and 1.33 , respectively (21); therefore, it is conceivable that the CaP crystalline deposition observed in the present study was a mixture of several CaP crystals.
In the present study, mouse MC3T3-E1 preosteoblast cells were used to investigate the proliferation, adhesion and expression of bone-related protein, according to a previous report (22). The cell proliferation results were presented as OD values measured in an MTT assay (Fig. 3). The OD value increased with time in the control and BM groups. In addition, SEM images revealed that the MC3T3-E1 cells successfully attached and spread on the PET and BM sheets after seven days of culture (Fig. 4), with a significant amount of extracellular matrix covering the fibers of the sheets. Previous studies have demonstrated that the biomimetic $\mathrm{CaP}$ layer supports the proliferation and adhesion of osteoblasts for long culture periods (23-25). Furthermore, MC3T3-E1 cells have been demonstrated to preferentially adhere to and/or proliferate in regions with a high CaP content (26). Notably, the BM group were observed to have a lower optical density $(0.160 \pm 0.022)$ compared with the control group $(0.196 \pm 0.014)$ after seven days of cell culture. Considering that the BM group had a significantly higher ALP activity compared with the control group (Fig. 5), the lower proliferation observed may have been the result of the induction of differentiation stimulated by the biomimetic $\mathrm{CaP}$ coating $(27,28)$.

In conclusion, a biomimetic $\mathrm{CaP}$ coating was successfully prepared on a PET artificial ligament in the present study. The organic, multiple layers of hyaluronic acid and CS functioned as an effective and efficient template to induce the deposition of nanoscale $\mathrm{CaP}$ apatites. In addition, the biomimetic $\mathrm{CaP}$ coating had a superior property with regard to the promotion of osteoblast activity in vitro. However, further studies are being undertaken to investigate the effects of different amounts of this biomimetic CaP coating on the PET ligament surface in vivo.

\section{Acknowledgements}

The study was supported by grants from the 973 Project (no. 2009CB930000) of the Ministry of Science and Technology of China and the Nano Project of Shanghai Municipal Science and Technology Commission (no. 1052nm03701). 


\section{References}

1. Nau T, Lavoie P and Duval N: A new generation of artificial ligaments in reconstruction of the anterior cruciate ligament. Two-year follow-up of a randomised trial. J Bone Joint Surg Br 84: 356-360, 2002.

2. Machotka Z, Scarborough I, Duncan W, Kumar S and Perraton L: Anterior cruciate ligament repair with LARS (ligament advanced reinforcement system): a systematic review. Sports Med Arthrosc Rehabil Ther Technol 2: 29, 2010.

3. Kock HJ, Stürmer KM, Letsch R and Schmit-Neuerburg KP: Interface and biocompatibility of polyethylene terephthalate knee ligament prostheses. A histological and ultrastructural device retrieval analysis in failed synthetic implants used for surgical repair of anterior cruciate ligaments. Arch Orthop Trauma Surg 114: 1-7, 1994.

4. Guidoin MF, Marois Y, Bejui J, Poddevin N, King MW and Guidoin R: Analysis of retrieved polymer fiber based replacements for the ACL. Biomaterials 21: 2461-2474, 2000.

5. Li H, Chen S, Wu Y, et al: Enhancement of the osseointegration of a polyethylene terephthalate artificial ligament graft in a bone tunnel using 58S bioglass. Int Orthop 36: 191-197, 2012.

6. Gao K, Chen S, Wang L, et al: Anterior cruciate ligament reconstruction with LARS artificial ligament: a multicenter study with 3- to 5-year follow-up. Arthroscopy 26: 515-523, 2010.

7. Xu L, Pan F, Yu G, Yang L, Zhang E and Yang K: In vitro and in vivo evaluation of the surface bioactivity of a calcium phosphate coated magnesium alloy. Biomaterials 30: 1512-1523, 2009.

8. Goyenvalle E, Aguado E, Nguyen JM, et al: Osteointegration of femoral stem prostheses with a bilayered calcium phosphate coating. Biomaterials 27: 1119-1128, 2006.

9. de Jonge LT, Leeuwenburgh SC, van den Beucken JJ, et al: The osteogenic effect of electrosprayed nanoscale collagen/calcium phosphate coatings on titanium. Biomaterials 31: 2461-2469, 2010.

10. Bigi A, Fini M, Bracci B, et al: The response of bone to nanocrystalline hydroxyapatite-coated Ti13Nb11Zr alloy in an animal model. Biomaterials 29: 1730-1736, 2008.

11. Lui P, Zhang P, Chan K and Qin L: Biology and augmentation of tendon-bone insertion repair. J Orthop Surg Res 5: 59, 2010.

12. Baxter FR, Bach JS, Detrez F, et al: Augmentation of bone tunnel healing in anterior cruciate ligament grafts: application of calcium phosphates and other materials. J Tissue Eng 2010 712370, 2010.

13. Mutsuzaki H, Sakane M, Fujie H, Hattori S, Kobayashi H and Ochiai N: Effect of calcium phosphate-hybridized tendon graft on biomechanical behavior in anterior cruciate ligament reconstruction in a goat model: novel technique for improving tendon-bone healing. Am J Sports Med 39: 1059-1066, 2011.
14. Mutsuzaki H, Sakane M, Nakajima H, et al Calcium-phosphate-hybridized tendon directly promotes regeneration of tendon-bone insertion. J Biomed Mater Res A 70: 319-327, 2004.

15. Li H, Ge Y, Wu Y, et al: Hydroxyapatite coating enhances polyethylene terephthalate artificial ligament graft osseointegration in the bone tunnel. Int Orthop 35: 1561-1567, 2011.

16. Li H, Ge Y,Zhang P, Wu L and Chen S: The effect of layer-by-layer chitosan-hyaluronic acid coating on graft-to-bone healing of a poly(ethylene terephthalate) artificial ligament. J Biomater Sci Polym Ed 23: 425-438, 2012.

17. Supová M: Problem of hydroxyapatite dispersion in polymer matrices: a review. J Mater Sci Mater Med 20: 1201-1213, 2009.

18. Boanini E, Torricelli P, Gazzano M, Giardino R and Bigi A: Nanocomposites of hydroxyapatite with aspartic acid and glutamic acid and their interaction with osteoblast-like cells. Biomaterials 27: 4428-4433, 2006.

19. Miyazaki T, Ohtsuki C, Akioka Y, et al: Apatite deposition on polyamide films containing carboxyl group in a biomimetic solution. J Mater Sci Mater Med 14: 569-574, 2003.

20. Zhang LJ, Liu HG, Feng XS, et al: Mineralization mechanism of calcium phosphates under three kinds of Langmuir monolayers. Langmuir 20: 2243-2249, 2004.

21. Barrère F, van Blitterswijk CA and de Groot K: Bone regeneration: molecular and cellular interactions with calcium phosphate ceramics. Int J Nanomedicine 1: 317-332, 2006.

22. Ku Y, Chung CP and Jang JH: The effect of the surface modification of titanium using a recombinant fragment of fibronectin and vitronectin on cell behavior. Biomaterials 26: 5153-5157, 2005.

23. Li X, Xie J, Yuan X and Xia Y: Coating electrospun poly(epsilon-caprolactone) fibers with gelatin and calcium phosphate and their use as biomimetic scaffolds for bone tissue engineering. Langmuir 24: 14145-14150, 2008.

24. Araujo JV, Martins A, Leonor IB, Pinho ED, Reis RL and Neves NM: Surface controlled biomimetic coating of polycaprolactone nanofiber meshes to be used as bone extracellular matrix analogues. J Biomater Sci Polym Ed 19: 1261-1278, 2008.

25. Arafat MT, Lam CX, Ekaputra AK, Wong SY, Li X and Gibson I: Biomimetic composite coating on rapid prototyped scaffolds for bone tissue engineering. Acta Biomater 7: 809-820, 2011.

26. Li X, Xie J, Lipner J, Yuan X, Thomopoulos S and Xia Y: Nanofiber scaffolds with gradations in mineral content for mimicking the tendon-to-bone insertion site. Nano Lett 9: 2763-2768, 2009.

27. Liu X, Smith LA, Hu J and Ma PX: Biomimetic nanofibrous gelatin/apatite composite scaffolds for bone tissue engineering. Biomaterials 30: 2252-2258, 2009.

28. Mavis B, Demirtas TT, Gümüşderelioĝlu M, Gündüz $\mathrm{G}$ and Colak U: Synthesis, characterization and osteoblastic activity of polycaprolactone nanofibers coated with biomimetic calcium phosphate. Acta Biomater 5: 3098-3111, 2009. 